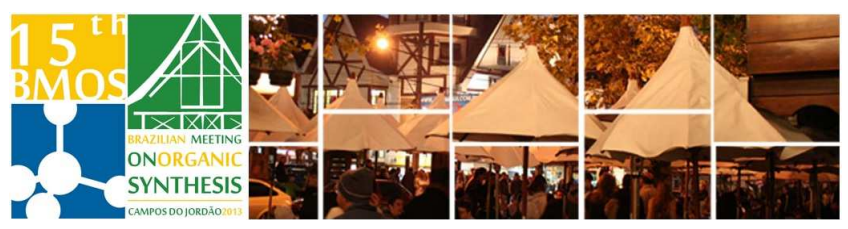

\title{
On the Biginelli Reaction under Homogeneous Catalysis
}

\author{
Haline G. O. Alvim, ${ }^{a}$ Tatiani B. de Lima, ${ }^{b}$ Heibbe C. B. Oliveira, ${ }^{a}$ Fabio C. Gozzo, ${ }^{b}$ \\ Julio L. Macedo, ${ }^{a}$ Patricia V. Abdelnur, ${ }^{\mathrm{c}}$ Wender A. Silva ${ }^{\mathrm{a}}$ and Brenno A. D. Neto ${ }^{\mathrm{a}}$
}

a) Institute of Chemistry, IQ-UnB, University of Brasilia, 70904-970, P.O.Box 4478, Brasília, Brazil b) Institute of Chemistry, University of Campinas. c) Embrapa Agroenergia, Brasília, Brazil.

*e-mail corresponding author: haline.alvim@gmail.com

Keywords: Biginelli reaction, ionic liquids, mechanism

\section{INTRODUCTION}

Today, new and greener technologies are imperative needs regarding the chemical industries. Attempts to connect the advantages of catalysis in ionic liquids $(\mathrm{ILs})^{1}$ with multicomponent (MCRs) reactions are found. $^{2}$ The Biginelli MCR (Scheme 1) was therefore performed with an efficient new homogenous catalytic system towards DHPM synthesis in ILs with an imidazolium Bronsted IL bearing $\left[\mathrm{PW}_{12} \mathrm{O}_{40}\right]^{3-}$.
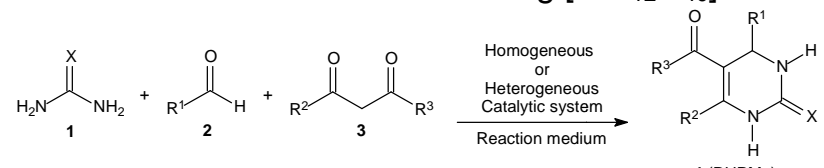

Scheme 1. General catalyzed Biginelli reaction.

\section{RESULTS AND DISCUSSION}

Scheme 2 shows the catalyst synthesis, which was further supported in BMI.NTf $\mathrm{NT}_{2}$ for the reaction.
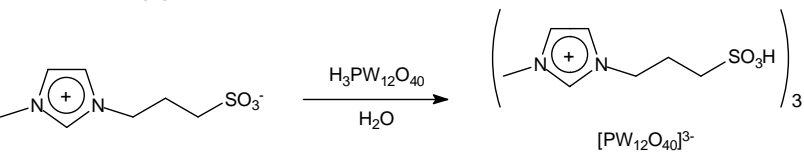

(MSI) ${ }_{3} \mathrm{PW}$

Scheme 2. Synthesis of the homogeneous catalyst (MSI) $)_{3} \mathrm{PW}$

Fifteen different DHPMs, including the bioactive Monastrol, were synthesized in good to excellent yields (60-99\%). To investigate the mechanism, ESIQTOF-MS analyses were performed (Figure 1).
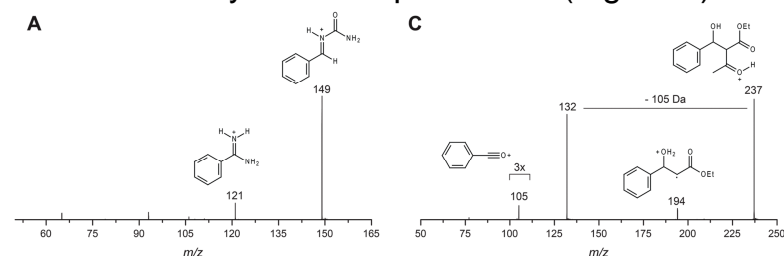

B

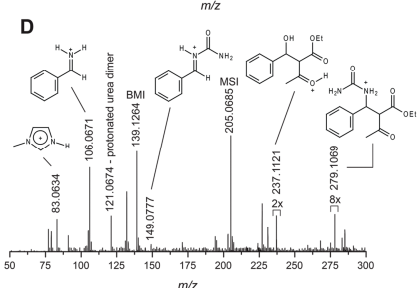

Figure 1. (A)-(C) ESI(+)-QTOF of the key intermediates.(D) ESI(+)-QTOF mass spectrum of the reaction.
The iminium mechanism was clearly the preferred reaction pathway as indicated by the detection of the iminium intermediate (Int I, Figure 1A) and the intermediate from the addition reaction to this ion (Int II, Figure 1B).

Finally, to gain insights into the mechanism and mainly into the IL effect, DFT calculations were also performed (Figure 2) considering the anion $\left(\mathrm{NTF}_{2}\right)$ effect over the charged cationic intermediates detected by ESI-MS analyses.
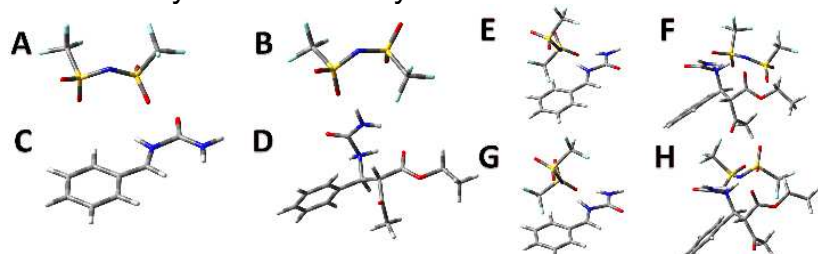

Figure 2. Optimized geometries at $B 3 L Y P / 6-31+G(d, p)$ level of theory. (A) Anion ( $\mathrm{NTf}_{2}$ ) cis. (B) Anion ( $\mathrm{NTf}_{2}$ ) trans. (C) Int I (iminium). (D) Int II (addition to the iminium). (E) Int I associated with the anion $\mathrm{NTf}_{2}$. Note the anion changed to its trans conformation upon approximation. (F) Int II associated with the anion $\mathrm{NTf}_{2}$ (cis). (G) Int I associated with the anion $\mathrm{NTf}_{2}$ (trans). (H) Int II associated with the anion $\mathrm{NTf}_{2}$ (trans).

The anion $\left(\mathrm{NTf}_{2}\right)$ from the IL (reaction medium) stabilized the charged (cationic) key intermediates upon ion pairing (and larger supramolecular aggregates) formation.

\section{CONCLUSION}

The efficiency of the new homogeneous catalytic system $\left((\mathbf{M S I})_{3} \mathbf{P W} /\right.$ BMI.NTf $\left._{2}\right)$ to perform the Biginelli MCR with enhanced IL effect was demonstrated. ${ }^{3}$ ESI-MS analyses and DFT calculations pointed the preferred mechanism pathway (iminium) and the origin of the IL effect through both ion pairing and larger supramolecular aggregates formation.

\section{ACKNOWLEDGEMENTS}

CAPES, CNPq, FAPDF, FAPESP and DPP-UnB.

\section{REFERENCES}

Dupont, J.: Souza, R. F.; Suarez, P. A. Z. Chem. Rev. 2002, 102, 3667.

de Graaff, C.; Ruijter, E.; Orru, R. V. A. Chem. Soc. Rev. 2012, 41, 3969.

Alvim, H. G. O.; de Lima, T. B.; de Oliveira, H. C. B.; Gozzo, F. C.; de Macedo, J. L.; Abdelnur, P. V.; Silva, W. A.; Neto, B. A. D. ACS Catal. 2013, 3, 1420 